\title{
EXEMPLES DE REGISTRES DE LANGUE DANS LA COMÉDIE ET LA TRAGÉDIE ET LEUR INTRODUCTION EN CLASSE DE FLE
}

\author{
Hanife Nâlân GENÇ ${ }^{1}$ \\ Songül Erdoğan GÜVEL $\dot{I}^{2}$
}

"Je tiens ce monde pour ce qu'il est: un théâtre où chacun doit jouer son rôle." William Shakespeare "La comédie corrige les manières, et le théâtre corrompt les mœurs." Louis de Bonald "Le sujet d'une belle tragédie doit n'être pas vraisemblable."

Corneille

Öz: Dil düzeyi alıcıya kendimizi ifade etme biçimidir. Teklifsiz, günlük ve üst düzey dil kullanımı olmak üzere başlıca üç temel ulamdan oluşur. Söz dağarcığı, yazım, dilbilgisi, deyimler, sözdizimi hatta fiil çekimi dil düzeylerine bağlı olarak değişim gösterir. Çalışmamızda, dil düzeylerini, içeriklerindeki katı teatral kurallara uymanın uzun bir süre geçerli olduğu klasik iki tür olan komedi ve trajedide inceledik. Söz konusu yapitlar gözlemlerimizi güçlendirmişlerdir. Komedi türünde Molière'in biri sadakatsiz bir kişiliği konu alan Dom Juan, diğeri kızların eğitimi üzerine yazılmış olan Les Femmes Savantes isimli iki tiyatro oyunu üzerinde durduk. Klasik trajedi türünde ise tercihimiz Corneille'in Le Cid'i ile Racine'in Phèdre'i yönünde olmuştur. Çalışmamızda komedide dil düzeylerini incelemeden önce tiyatroda oldukça baskın bir niteliğe sahip olan dil düzeyleri ve dolaylı olarak da sahnede kullanımları üzerinde duracağız. Araştırmamızı klasik trajedide kullanılan dil ile tamamlayacağız. Bu yolla yabancı dil olarak Fransızcanın öğretiminde dil kazanımının farklı dil düzeylerinde nasıl gerçekleştirilebileceğine ilişkin kimi öneri ve yaklaşımlarda da bulunabilme olanağına sahip olacağız. Böyle bir yaklaşım yalnızca dört temel dil becerisinin öğretiminde değil, aynı zamanda söz dağarcığı, dilbilgisi, ses bilgisi öğretiminde de etkin olacaktır. Öğrenen, sözdizimsel ve dilbilgisel yetilerini geliştirmekle

\footnotetext{
${ }^{1}$ Prof. Dr., Ondokuz Mayıs Üniversitesi, Eğitim Fakültesi,Yabancı Diller Eğitimi Bölümü, Fransız Dili Eğitimi Anabilim Dalı. ngenc@omu.edu.tr

${ }^{2}$ Yüksek Lisans Öğrencisi, Ondokuz Mayıs Üniversitesi, Eğitim Bilimleri Enstitüsü, Yabanc1 Diller Eğitimi Bölümü, Fransız Dili Eğitimi Anabilim Dalı. ssg.erdogan@gmail.com
} 
kalmayıp çoğunlukla güçlük yaşanan pragmatik ve anlambilimsel düzlemde de gelişim kaydedebilecektir.

Anahtar Sözcükler: Fransızca Öğretimi, Dil Düzeyi, Teklifsiz, Günlük ve Üst Düzey Dil Kullanımı, Kökleşik Tiyatro, Komedi, Trajedi.

\section{Introduction}

Le registre de langue que l'on connait sous d'autres appellations comme niveau de langue ou style de langue peuvent nous orienter vers différentes impressions. De ce point de vue nous pouvons considérer le mot 'registre' comme un terme plus ou moins synonyme de style. Le terme 'niveau de langue' a plutôt une relation avec la structure grammaticale. Quant au terme 'style de langue' il nous renvoie à la stylistique du langage et est plutôt en rapport avec la littérature. "Les créations littéraires ne nous seraient pas accessibles si elles n'entraient pas en partie, par les moyens d'expression que nous comprenons et que nous employons sans cesse" (Bally, 1951, p. 181). En effet, nous avons choisi d'adopter le terme registre de langue au lieu du terme style et niveau de langue car cette expression englobe les deux autres à la fois, nous paraît-il. Dans une situation de communication que ce soit les expressions lexicales ou les expressions syntaxiques, celles-ci se transmettent de façon très variées. Pour cette raison, le registre de langue a un lien étroit avec le vocabulaire, l'orthographe, la grammaire et la conjugaison puisque ses éléments changent lorsque le niveau de langage change. Le niveau de langue grosso modo est "une autre manière de voir les mêmes choses, une autre manière de les décrire et de les interpréter" (Benveniste, 1974, p. 79).

De nos jours, les approches ou méthodes de l'enseignement d'une langue étrangère accordent beaucoup d'attention à l'apprentissage de tous les aspects. Lorsque que l'on regarde de cette perspective, c'est la langue dans sa totalité et sa forme multidimensionnelle qui est prise en considération. Vu que "la langue française n'est pas un bloc homogène" (Homsi, 2005, p. 34) celle-ci doit être considérée dans tous ses aspects. L'apprenant prendra plaisir dans cet enseignement des divers registres de langue en découvrant chaque fois la profondeur de toutes les composantes de la langue comme la stylistique, le lexique, l'orthographe, la syntaxe et même la phonologie. D'ailleurs, dans les nouvelles approches de l'enseignement d'une langue étrangère, l'objectif n'est plus de se limiter à un langage standard que l'on retrouve dans les livres de lecture ou les manuels scolaires. L'apprenant peut déduire qu'il ne maîtrise pas la langue apprise puisqu'elle ne sera pas fonctionnelle dans sa vie de tous les jours. Certes, l'acquisition des différents registres tels que le familier, le courant ou le soutenu lui sera plus enrichissante. À noter que ces trois derniers lui seront nécessaires dans différents états ou situations. Peut-on affirmer qu'un apprenant ne sachant pas que le mot "dodo" signifie "dormir" dans le langage enfantin et familier, maîtrise parfaitement la langue? Ou si l'on devrait regarder d'un autre point de vue, le mot 'vélocité' désignant 'rapidité' sera encore une fois un obstacle dans la compréhension de l'apprenant. Nous pouvons ajouter même qu'un débutant découvrant le mot 'vélo' ne pourra peut-être pas mettre en 
relation ces deux mots. Si l'on devrait prendre en compte toutes les raisons citées ci-dessus, tout d'abord il convient de ne pas se restreindre aux cours. Le premier objectif influent n'est plus de se délimiter seulement aux connaissances en classe mais plutôt les transporter à la vie c'est-à-dire de les apprendre en vivant. 'Apprendre en vivant' nous oriente vers l'improvisation, la simulation, la dramatisation, la spontanéité, l'animation, l'imagination etc. Étant donné que les jeux de rôles ont tous une affinité avec le théâtre, l'intégration du théâtre en milieu scolaire apporterait non seulement une richesse esthétique mais aussi un apprentissage permanent pour l'apprenant. Il s'agirait d'un moment où chacun voudrait trouver sa place dans une telle atmosphère. Dans cette perspective, nous pensons que les activités théâtrales en milieu scolaire peuvent être non seulement une approche dynamique mais aussi un bon outil pédagogique d'enseignement pour l'apprenant ainsi que pour l'enseignant. Lorsqu'il s'agit du théâtre, les premiers genres venant à l'idée sont sans aucun doute, la tragédie et la comédie. Pour cette raison, il serait donc intéressant de connaître le registre de langue dans une comédie et une tragédie classiques. Afin d'expliciter notre étude suite à des extraits d'œuvres analysés, nous pourrons même déduire à la fin de notre étude quel type de pièce de théâtre serait convenable dans une classe d'enseignement de langue française puisque le théâtre est ludique, pratique et à la fois instructif.

\section{Les registres de langue}

Comme nous le savons, la langue ne s'utilise pas de la même façon dans tous les actes de parole. Pour cela, un mot peut être doté de plusieurs et différents sens selon son utilisation et le contexte: le sexe, le statut social, l'âge, le niveau de culture, la situation dans laquelle les interlocuteurs se trouvent, la relation ou le niveau de connaissance entre ceux-ci, leur intention et leur objectif donnent un sens à l'acte de parole. Le registre de langue renvoie au terme 'style' qui désigne l'utilisation cohérente et élitiste du langage. Le mot style "impliqué par la notion de manière" (Dessons, 2004, p. 101) englobe non seulement la sphère de la littérature mais encore celle de l'art. D'un autre point de vue, le style entretient un rapport avec la grammaire et la stylistique et il est souvent difficile de les détacher. La frontière entre les deux n'est pas décisive. "Cette frontière est instable dans la vie même de la langue, où certaines formes se trouvent dans un processus de grammaticalisation, tandis que d'autres sont en cours de dégrammaticalisation" (Bakhtine-Volochinov, 1977, p. 174). La préférence du niveau entraîne avec elle une certaine subjectivité car le style devient "marque de l'individu". (1). Cette marque peut être appréciée comme "une manière d'écrire propre à une personne" (2).

Le registre de langue se réalise au niveau de la syntaxe, de la grammaire et du vocabulaire utilisés et il peut différer selon l'interlocuteur; un ami, un membre de famille ou un supérieur hiérarchique. Nous pouvons parler de trois stylistiques: "descriptive, fonctionnelle, génétique"(Guiraud, 1969, p. 27). Mais nous allons laisser d'un côté la stylistique pour nous orienter plutôt vers le niveau de langue. Il existe essentiellement trois formes de niveau de langue. 


\subsection{Le registre familier}

Le langage familier comme son nom l'indique est très souvent employé entre des membres de famille, des amis, des collègues de travail, des camarades de classe c'est-à-dire des personnes que l'on connaît. Le vocabulaire est 'relâché' et parfois même abrégé (on peut rencontrer des mots dits à l'envers comme par exemple ci-mer au lieu de merci). Quant aux règles de grammaire, elles ne sont pas toujours respectées. Plus souvent utilisé à l'oral, dans le registre familier toutes les syllabes ne sont pas employées. Ce registre peut être utilisé pour plusieurs raisons; sous prétexte qu'elle est plus pratique et plus économique et montre un lien plus rapproché entre les interlocuteurs.

Exemples:

J't'avais pas dit de faire gaffe pour je t'avais demandé de faire attention!

Ta vu mes pompes pour tu as vu mes chaussures.

\subsection{Le registre courant}

Le langage courant qui est le plus souvent utilisé dans la vie de tous les jours correspond à un langage correct. Les règles de syntaxe et la grammaire sont plutôt respectées et le vocabulaire employé à l'oral est convenable pour l'écrit. Dans les échanges de type professionnel, officiel, administratif et commercial ou quand la communication se déroule entre des interlocuteurs qui ont une certaine distance, le langage courant est celui qui est le mieux adapté.

Exemples:

Je suis indisponible pour le moment.

Les enfants sont très bruyants.

\subsection{Le registre soutenu}

Le langage soutenu, aussi appelé soigné, est employé dans la littérature, surtout dans la poésie et la tragédie. Elle nécessite le bon usage. Dans les deux derniers que nous venons de citer, celui-ci est le registre sublime qui va jusqu'au sens d'apothéotique. Ce registre s'évalue pour deux raisons: avec la préférence du vocabulaire assez recherché et avec de la grammaire assez bien déterminée. La formation des propositions aussi se différencie avec leur longueur. Cette longueur appelée aussi période peut avoir de différente forme. La syntaxe complexe dans ces phrases peut être allongée sur une dizaine de lignes.

Je me suis tellement accoutumé ces jours passés à détacher mon esprit des sens, et j'ai si exactement remarqué qu'il y a fort peu de choses que l'on connaisse avec certitude touchant les choses corporelles, qu'il y en a beaucoup plus qui nous sont connues touchant l'esprit humain, et beaucoup plus encore de Dieu même, qu'il me sera maintenant aisé de détourner ma pensée de la considération des choses sensibles ou imaginables, pour la porter à celles qui, étant dégagées de toute matière, sont purement intelligibles. (Descartes-Méditation quatrième).

Dans le registre soutenu le vocabulaire et les figures de style sont assez recherchés. L'azur ou les cieux pour le ciel peut être un exemple. "Dans les temps de l'écrit c'est l'imparfait et le plus-que-parfait du subjonctif qui sont 
utilisés (exemple: Il fallait qu'il vînt pour Il fallait qu'il vienne). A l'oral nous retrouvons le passé simple et le passé antérieur de l'indicatif (exemple: je le vis quand je revins pour Je l'ai vu quand je suis revenu.)" (3).

Apres avoir examiné les registres de langue en français il serait intéressant de savoir lequel des trois est utilisé dans la comédie. Pour cela, nous illustrerons nos exemples par le biais de deux œuvres de Molière: Les Femmes Savantes et Dom Juan.

\section{La comédie}

Tout d'abord, il ne faut pas confondre le comique qui est un registre littéraire et la comédie qui est un genre. Mais tous deux proviennent du mot grec kômos signifiant une procession festive en l'honneur de Dionysos, Dieu de la vigne et du vin mais aussi père du théâtre et de la tragédie (4). Le registre comique vise à faire rire et à divertir le spectateur. Il peut aussi avoir une fonction critique sur les hommes, sur la société ou sur le comportement d'une catégorie de personnes.

\subsection{Les différents types de registres comiques}

Si l'on devrait parler en quelques mots des registres, il en existe trois: le registre satirique qui se moque des défauts d'une personne ou d'une catégorie de personnes. Le registre parodique ayant pour but de faire rire. C'est une imitation d'un texte par exemple. Enfin, le registre burlesque qui est le traitement d'un sujet sérieux en style vulgaire. Le burlesque repose sur le contraste entre le style et le sujet traité.

\subsection{Les différents types de comédie}

Dans la comédie française, nous retrouvons quatre styles de comédie. La comédie de mœurs d'abord, c'est un type de comédie du XVIème siècle qui dénonce les excès d'une société ou d'un groupe de personnes. Quant à la comédie de caractère, c'est une satire qui met en avant les défauts d'une société. La comédie d'intrigue comme son nom l'indique est un genre théâtral ludique $\mathrm{du}$ fait de l'abondance des actions et rebondissements qu'il propose. Enfin la comédie de sentiments aussi appelée comédie héroïque est un style qui décrit souvent les exploits d'un personnage animé par le sentiment de l'amour (5).

\subsection{Le registre de langue dans une comédie théâtrale}

Nous verrons dans cette partie les caractéristiques dans une comédie grâce à deux œuvres de Molière. Nous avons choisi ces extraits car ces deux scènes typiques mettent en avant l'usage des registres de langue. Dans Dom Juan, acte II scène 3, Dom Juan, le protagoniste, Sganarelle, son valet, Pierrot, un paysan et Charlotte une paysanne conversent. Pierrot d'abord entre en dialogue avec Dom Juan et Charlotte s'incruste. Ils parlent très mal le français. À cette époque, la quasi-totalité des paysans étaient analphabètes et donc parlaient la langue d'après ce qu'ils entendaient autour d'eux. Cet extrait du dialogue entre Pierrot et Charlotte le démontre bien: 
Pierrot - Testigenne! Parce qu'ous estes Monsieu, ous viendrez caresser nos femmes à notre barbe ? Allez-v's-en caresser les vôtres (...)

Charlotte - Va, va, Piarrot, ne te mets point en peine : si je sis Madame, je te ferai gagner queuque chose, et tu apporteras du beurre et du fromage cheux nous.

Pierrot - Ventrequenne ! Je gni en porterai jamais, quand tu m'en poyrais deux fois autant. Est-ce donc comme ça que t'écoutes ce qu'il te dit ? Morquenne ! Si j'avais su tantost, je me serais bian gardé de le tirer de gliau, et je gli aurais baillé (donné) un bon coup d'aviron sur la teste.

Le langage est familier. Les personnages ont un vocabulaire très restreint et font beaucoup de fautes à l'oral. Cela montre que c'est un milieu modeste où les gens ne savent ni lire et ni écrire. Plus loin dans l'acte II scène 4 nous rencontrons encore une conversation entre deux paysannes; Charlotte et Mathurine. Dom Juan a promis à chacune qu'il allait l'épouser. Encore une fois nous apercevons le niveau de langue médiocre.

Charlotte - Vous voyez qu'al le soutient.

Dom Juan, bas, a Charlotte. - Laissez-la faire.

Mathurine - Vous êtes témoin comme al l'assure.

Dom Juan, bas, a Mathurine. - Laissez-la faire. (...)

Mathurine - Oui, Charlotte, je veux que Monsieur vous rende un peu camuse.

Charlotte - Monsieur, vuidez la querelle, s'il vous plaît.

Dans le début de cette comédie nous rencontrons plusieurs dialogues assez comiques entre les paysans, Charlotte-Pierrot et Charlotte-Mathurine. Cela montre que ces personnages viennent d'un milieu populaire et ils parlent comme à un membre de la famille ou un ami sans faire attention à la construction de la phrase (la syntaxe) et au vocabulaire (le lexique). C'est le langage familier qui est utilisé dans ces conversations.

Nous pouvons examiner de près une autre œuvre de Molière qui est aussi une comédie, Les Femmes Savantes. Dans cette œuvre nous retrouvons le comique de langage comme dans Dom Juan. En effet, ce n'est pas seulement par l'analphabétisme de paysans que Molière met en avant le comique de langage mais aussi par l'exagération du langage soutenu utilisé par les personnages. Tout d'abord dans 1'acte II scène 6 la conversation entre Martine, la servante de cuisine, Bélise, la sœur de Chrysale et Philaminte, la femme de Chrysale. Philaminte et Bélise pourchassent Martine avec pour motif la mauvaise utilisation du vocabulaire et de la grammaire. C'est une faute lourde aux yeux de Philaminte et Bélise.

Martine - Mon Dieu, je n'avons pas étugué comme vous, Et je parlons tout droit comme on parle cheux nous.

Philaminte - Ah, peut-on y tenir !

Bélise - Quel solécisme horrible !

Philaminte - En voilà pour tuer une oreille sensible. 
Bélise - Ton esprit, je l'avoue, est bien matériel. « Je » n'est qu'un singulier ; « avons » est pluriel. Veux-tu toute ta vie offenser la grammaire ?

Martine - Qui parle d'offenser grand-mère, ni grand-père?

Philaminte - Ô Ciel !

Bélise - "Grammaire" est prise à contresens par toi, Et je t'ai déjà dit d'où vient ce mot.

Martine - Ma foi, Qu'il vienne de Chaillot, d'Auteuil, de Pontoise, cela ne me fait rien.

Bélise - Quelle âme villageoise ! La grammaire, du verbe et du nominatif, comme de l'adjectif avec le substantif, nous enseigne les lois.

Martine - J'ai, Madame, a vous dire que je ne connais point ces gens-là.

Dans ce dialogue nous pouvons observer le langage populaire de Martine avec des fautes d'orthographe et de vocabulaire. C'est une forme de langage que nous trouvons dans toutes les comédies de Molière. C'est une façon de dénoncer les excès d'une société. Par ailleurs, dans l'acte III scène 3, la conversation élogieuse entre Vadius et Trissotin commence avec un vouvoiement. Ils parlent en vers et leur propos sont assez flatteurs. Le registre de langue est mal adapté à la situation et les phrases n'ont pas de sens. C'est une autre forme de la comédie. Pour Vadius et Trissotin, il faut être cultivé et savoir le montrer en parlant dans un langage soutenu.

Trissotin - Aux ballades surtout vous êtes admirable.

Vadius - Et dans les bouts-rimés je vous trouve adorable.

Trissotin - Si la France pouvait connaître votre prix,

Vadius - Si le siecle rendait justice aux beaux exprits,

Trissotin - En carrosse doré vous iriez par les rues.

Vadius - on verrait le public vous dresser des statues. Hom. C'est une ballade, et je veux que tout net Vous m'en...

Mais ce dialogue continue par un tutoiement et le registre de langue change. Les deux deviennent plus agressifs. Leurs vers banals se complimentant réciproquement se traduisent en une querelle. Ils sont assez grossiers dans leurs paroles.

Trissotin - Allez, petit grimaud, barbouilleur de papier.

Vadius - Allez, rimeur de balle, opprobre du métier.

Trissotin - Allez, fripier d'écrits, impudent plagiaire.

Vadius - Allez, cuistre...

Nous remarquons dans cette comédie qu'il y'a un jeu sur le registre de langue. Molière exagère dans le langage soutenu mais lorsque les personnages reviennent à eux-mêmes ils commencent à parler dans le langage familier voire populaire puisqu'ils sont assez grossiers dans leurs phrases.

Apres avoir étudié le langage dans la comédie théâtrale nous verrons dans cette dernière partie le registre de langue dans une tragédie. Pour cela nous nous 
servirons de deux œuvres; l'une de Racine Phèdre et l'autre de Corneille Le Cid.

\section{La tragédie («Phèdre» de Racine et «Le Cid» de Corneille)}

Le tragique est un registre et la tragédie est un genre théâtral. "Depuis la Poétique d'Aristote, les genres théâtraux classiques se définissent traditionnellement par la classe sociale des personnages principaux, par le type d'obstacles qu'ils rencontrent dans l'intrigue de la pièce et par la réaction des spectateurs" (6). Très souvent dans une tragédie nous retrouvons un héros ou des personnages de rang social élevé. La tragédie met en scène une haute noblesse qui se heurte à la fatalité et suscite " l'admiration et la crainte" (7). L'objectif étant d'émouvoir et d'instruire le spectateur, le dramaturge veut provoquer la pitié chez le spectateur. "Dominée par les registres tragique, pathétique, épique ou lyrique, la tragédie vise à provoquer l'admiration et la pitié chez les spectateurs" (Pagès, 2001). D'ailleurs, un texte tragique se termine souvent mal c'est-à-dire par la mort d'un personnage ou par une folie.

\subsection{La situation tragique et les règles à respecter}

La tragédie classique a une progression dramatique qu'il serait intéressant de rappeler avant d'étudier une œuvre dramatique. Elle se compose d'une exposition, d'un nœud et d'un dénouement. L'exposition est le début de la pièce qui contient les éléments essentiels et tous les personnages que le spectateur rencontrera jusqu'à la fin de la pièce de théâtre. De plus, ce dernier doit avoir connaissance des personnages, des relations ou liens entre eux, des lieux, de l'époque et de l'objet (l'intrigue). Ensuite, c'est le nœud qui est "composé selon Aristote, en partie de ce qui s'est passé hors du théâtre avant le commencement de l'action qu'on y décrit et en partie de ce qui s'y passe: le reste appartient au dénouement". C'est par ces paroles que Pierre Corneille définit le nœud dans la tragédie classique. Enfin c'est le dénouement où les problèmes exposés dans le nœud sont résolus. Cette fin se termine par la mort de quelqu'un.

\subsection{Le registre de langue dans une tragédie classique}

Afin d'illustrer notre étude sur le registre de langue utilisé dans la tragédie classique nous allons examiner d'abord l'œuvre de Corneille Le Cid qui a eu un immense succès en 1636. Dans une tragédie, les personnages s'expriment en vers (alexandrins). Dans l'acte I scène 1, Chimène, fille de Don Gomes s'exprime comme suit :

Dis-moi donc, je te prie, une seconde fois

Ce qui te fait jurer qu'il approuve mon choix ;

Apprends-moi de nouveau quel espoir j'en dois prendre ;

Un si charmant discours ne se peut trop entendre ;

Tu ne peux trop promettre aux feux de notre amour

La douce liberté de se montrer au jour.

Que t'a-t-il répondu sur la secrète brigue

Que font auprès de toi don Sanche et don Rodrigue ? 
N'as-tu point trop fait voir quelle inégalité

Entre ces deux amants me penche d'un côté ?"

Dans cet extrait nous voyons que les vers sont des alexandrins et ont une rime. Le registre de langue est soutenu. Cela est vu dans la construction des phrases et dans le vocabulaire choisi par Chimène comme "approuve mon choix", "la secrète brigue" ou la figure de style qui est aussi un élément du registre soutenu. Le personnage utilise la métaphore dans sa phrase "La douce liberté de se montrer au jour." Nous pouvons aussi citer un autre exemple dans le Cid encore avec les paroles de Don Rodrigue dans l'acte II scène 2 :

Oui ; tout autre que moi

Au seul bruit de ton nom pourrait trembler d'effroi.

Les palmes dont je vois ta tête si couverte

Semblent porter écrit le destin de ma perte.

J'attaque en téméraire un bras toujours vainqueur,

Mais j'aurai trop de force, ayant trop de cœur.

A qui venge son père il n'est rien d'impossible.

Ton bras est invaincu, mais non pas invincible.

Don Rodrigue comme Chimène a un langage sublime, le vocabulaire utilisé est assez recherché. Ici les mots choisis et la construction des phrases sont très correctes.

Nous pouvons analyser le langage de notre deuxième œuvre. Dans l'acte II scène 5 , Phèdre s'exclame :

Ah ! Cruel, tu m'as trop entendue.

Je t'en ai dit assez pour te tirer d'erreur.

Eh bien, connais donc Phèdre et toute sa fureur.

J'aime. Ne pense pas qu'au moment que je t'aime,

Innocente à mes yeux je m'approuve moi-même,

Ni que du fol amour qui trouble ma raison

Ma lâche complaisance ait nourri le poison.

Phèdre aussi s'exprime en alexandrins qui riment. Le langage de ce personnage ressemble à celui des personnages du Cid, c'est un langage soutenu "je m'approuve moi-même", "lâche complaisance". Le registre épique est utilisé dans ces vers que l'on peut voir avec les exclamations.

Noble et brillant auteur d'une triste famille

Toi, dont ma mère osait se vanter d'être fille,

Qui peut être rougis du trouble où tu me vois,

Soleil, je te viens voir pour la dernière fois.

Dieux ! que ne suis-je assise à l'ombre des forêts !

Quand pourrai-je, au travers d'une noble poussière,

Suivre de l'œil un char fuyant dans la carrière? 
Dans l'acte I scène 3, lorsque Phèdre s'adresse à Enone le langage soutenu est conservé : "noble et brillant", "ne suis-je assise", "un char fuyant". De même, une figure de style apparait. La métaphore du soleil. Ici le soleil sert à décrire le père de la famille. Nous pouvons voir ce type de conversation ou plutôt de vers dans toutes les tragédies classiques.

\section{Conclusion}

À travers notre étude, nous avons analysé les différences de registres de langue dans une comédie et une tragédie classiques avec quatre œuvres, aussi importante l'une que l'autre dans le théâtre français du XVIIème siècle. Comme nous l'avons indiqué au début de notre recherche, les registres de langue devront être appris dans leur profondeur pour une bonne connaissance langagière. Par la suite, la tragédie ainsi que la comédie trouveront place dans un milieu scolaire. Mais la fréquence de leur utilisation dépendra donc non seulement de l'intérêt des apprenants mais aussi de leur besoin langagier. Si l'on prend en considération ces deux aspects, il nous semble que l'apprenant s'orientera vers la comédie au lieu de la tragédie. La comédie divertit le spectateur, nous pouvons en-déduire que dans une classe d'enseignement de la langue française par exemple, elle conviendra mieux aux attentes de l'enseignant et de l'apprenant. Quant à la tragédie, celle-ci a un langage assez lourd qui relève de l'écrit et il serait difficile de l'exploiter dans une classe de français langue générale. D'ailleurs, nous l'avons souligné dans notre développement, c'est un langage utilisé dans la littérature, surtout dans la poésie. Pour rebondir sur la comédie, le registre de celle-ci est plutôt familier mais aussi courant. Il faudra faire attention lors du choix de la pièce de théâtre afin que celle-ci ne soit pas assez populaire dans son registre. Bien sûr, cela ne veut pas dire que la tragédie sera bannie et que la comédie sera le seul choix de l'enseignant. Ce dernier doit motiver l'apprenant à étudier la tragédie dans un concept intégral. D'ailleurs, dès que l'apprenant commencera à apprendre la langue par le biais du théâtre, autrement dit, une fois qu'il combinera la façon ludique et instructive, il prendra beaucoup de plaisir à apprendre. Il sera beaucoup plus motivé et plus ambitieux dans l'apprentissage de la langue étrangère dans toutes ses dimensions.

\section{Note}

(1) Dictionnaire de Rhétorique et de Poétiques, p. 353-356.

(2) Dictionnaire des Termes Littéraires, p. 457.

(3) voir,http://www.etudes-litteraires.com/figures-de-style/niveaux-delangage.php\#ixzz2mEtAtA95, (date de dernier accès 01/12/2013).

(4) http://www.etudes-litteraires.com/figures-de

style/tragedie.php\#ixzz2mFH5BLlg, (date de dernier accès 15/12/2013).

(5) www.comptoirlitteraire.com/.../372-marivaux-l... (date de dernier accès $12 / 12 / 2013)$.

(6) Genç, Hanife Nalan. Les notes du cours s'intitulant Les genres dramatiques et leur apprentissage donné dans le premier semestre de l'année 
scolaire 2013-2014 à l'Institut des Sciences Pédagogiques de l'Université Ondokuz Mayıs.

(7) Genç, Hanife Nalan. Cours sur le théâtre, 2013.

\title{
BIBLIOGRAPHIE
}

Bakhtine, M. M. et Volochinov, V. N. (1977). Le marxisme et la philosophie du langage essai d'application de la méthode sociologique en linguistique traduit du russe et présenté par Marina Yaguello; préface de Roman Jakobson. Paris: Minuit.

Bally, C. (1951). Traité de stylistique française. Paris: Klincksieck.

Benveniste, E. (1974). Problèmes de linguistique générale, II. Paris: Gallimard.

Corneille, P. Le Cid. Association de Bibliophiles Universels.

Dessons, G. (2004). L'Art et la manière-art, littérature, langage. Paris: Honoré Champion.

Dobransky, M. (1994). Les écrivains du Bac, La Tragédie classique. Paris: Gallimard.

Homsi, M. N. N. (2005). Langage et stylistique: Leçons de stylistique française. Guiraud, P. (1969). Essais de stylistique. Paris: Klincksieck.

Molière. (1665). Dom Juan. Paris: Hatier.

Molière. (1672). Les Femmes Savantes. Édition Le Livre de Poche.

Pagès, A. (Collectif) (2001). Français lycées. Méthodes et activités littéraires, programmes 2000-2001 Nouveau Bac. Paris: Nathan.

\section{EXAMPLES OF LANGUAGE REGISTERS IN COMEDY AND TRAGEDY AND THEIR USE IN FLE CLASSES}

\begin{abstract}
Language level is a way of expressing ourselves to the receiver. It consists of mainly three categories as colloquial, standard and formal language usage. Vocabulary, spelling, grammar, idioms, syntax even verb conjugations vary depending on the level. In our study we have examined comedy and tragedy, two classical genres known by their strict rules to which the playwrights obeyed for a long time. These works have strengthened our observations. In this essay we approach the two comedies written by Molière: Dom Juan about an unfaithful personality and Les Femmes Savantes written about girls' education. In classical tragedy genre, our preferences are Corneille's Le Cid and Racine's Phèdre. In our study before examining language levels in comedy genre, we primarily concentrate on language level which has a quite dominant nature in theatre genre and indirectly its usage on the stage. We complete our research with the language used in classical tragedy. Our object is to understand if it is possible to use these comedies and tragedies in order to teach different language levels. In this way, we will have the opportunity to suggest some ideas on how language acquisition will be realised at different language levels in FFL (French as a Foreign Language)
\end{abstract}


situations. Such an approach will be effective in the teaching of four main language skills, as well as vocabulary, grammar and pronunciation. The learner will thus develop not only his syntactic and grammatical competences, but also the pragmatic and semantic ones, which are more difficult to improve.

Keywords: Teaching French, Language Register, Colloquial, Standard and Formal Language Use, Classical Genres, Comedy, Tragedy. 